Article

\title{
Porous Organic Polymers-Supported Metallocene Catalysts for Ethylene/1-Hexene Copolymerization
}

\author{
Xiong Wang ${ }^{1, *}$, Xiaoyu Han ${ }^{1}$, Feng Ren ${ }^{1}$, Renwei $\mathrm{Xu}^{1}$ and Yongxiao Bai ${ }^{2, *}$ \\ 1 Lanzhou Petrochemical Research Center, Petrochemical Research Institute, PetroChina, Lanzhou 730060, \\ China; hanxiaoyu@petrochina.com.cn (X.H.); renfeng9@petrochina.com.cn (F.R.); \\ xurenwei@petrochina.com.cn (R.X.) \\ 2 School of Physical Science and Technology, Lanzhou University, Lanzhou 730000, China \\ * Correspondence: wangxiong1@petrochina.com.cn (X.W.); baiyx@lzu.edu.cn (Y.B.); \\ Tel.: +86-931-7983474 (X.W.); +86-931-8912406 (Y.B.); Fax: +86-931-7982766 (X.W.); +86-931-8913554 (Y.B.)
}

Received: 27 February 2018; Accepted: 2 April 2018; Published: 4 April 2018

\begin{abstract}
Porous organic polymers (POPs) have received much attention in adsorption, separation, and catalysis. In this paper, porous organic polymers with different pore structure were used as metallocene catalyst supports, and ethylene/1-hexene copolymerizations were conducted using the POPs-supported metallocene catalyst. The pore structure of the prepared POPs and the supported metallocene catalyst were characterized by nitrogen sorption porosimetry and non-local density functional theory simulation, and the molecular chain structure of the produced ethylene/1-hexene copolymers were investigated through gel permeation chromatography (GPC), IR analysis, differential scanning calorimetry (DSC), and temperature rising elution fractionation (TREF). The results show that the loading amount of active sites varied with different pore structures of the POP supports, and the active species scattered in different pore sizes had a moderate impact on the molecular chain growth and the molecular weight distribution. The IR, DSC, and TREF analysis revealed that different branching degree, double bond content, and chemical composition distributions were detected from the molecular chain structure of the ethylene/ $\alpha$-olefin copolymers from different POPs and silica-supported metallocene catalysts, despite their similar IR, DSC, and TREF curves due to the same active species. Scanning electron microscopy (SEM) showed that porous ethylene/ $\alpha$-olefin copolymers with varied surface morphology were obtained from the POPs-supported metallocene catalysts with different pore structure.
\end{abstract}

Keywords: porous organic polymer (POP); ethylene; 1-hexene; metallocene catalyst; copolymerization

\section{Introduction}

Since the discovery of catalysts for olefin polymerization by Ziegler, Natta, and Phillips in the 1950s, the production of polyolefins has continuously grown and the research in this field has remained very competitive [1]. The development of well-defined molecular catalysts giving access to fine control of the polymerization has allowed the preparation of polyolefins with improved properties and original materials [1,2]. Metallocene complexes have proved to be attractive catalysts for olefin polymerization, due to the possibility of influencing the catalyst activity and tailoring the properties of polyolefins such as the polymer molecular weight, comonomer incorporation, and stereospecificity by simply changing the structure of the ligands used [3].

In order to fulfill important requirements such as the control of product morphology, prevention of reactor fouling, and control of the catalyst cost for slurry or gas-phase process [4,5], immobilization of the metallocenes on suitable supports is necessary. Numerous studies on inorganic supports $\left(\mathrm{MgCl}_{2}, \mathrm{MgF}_{2}, \mathrm{Al}_{2} \mathrm{O}_{3}\right.$, molecular sieves, zeolite, and clays) and immobilization procedures have 
been investigated [6-14]. However, these supports suffer from several drawbacks, including the need for complex chemical treatments to get rid of acidic groups on their surfaces and to obtain appropriate particle morphology, and the presence of residual inorganic fragments within the produced polyolefins that may affect their mechanical and optical properties [15-19]. In contrast, porous organic polymer (POP) supports offer significant advantages over their inorganic equivalents: they provide a much closer analogue to the environment prevailing in homogeneous polymerization, do not require fastidious pre-treatment, and should not significantly affect the final polyolefin properties [9,20-22]. Furthermore, porous organic polymers can be properly designed and synthesized with tunable pore size distribution, flexible synthetic strategy, and readily modifiable functionality [23-31].

However, understanding structure/polyolefin properties relationships in these heterogeneous metallocene catalysts are nearly focused on metallocene complexes and activators, and little attention has been paid to the selection of supports for metallocene catalysts. In a previous work, we reported that the chain length of homo-polyethylene (PE) can be tuned by the pore structure of the nanoporous organic polymer supports [32]. Therefore, it is a simple, useful, and complementary method tothe metallocene complexes/activators systems to control the molecular structure of polyolefin - especially for ethylene / $\alpha$-olefin copolymers-by designing or tailoring the pore structure of the used POP supports. To obtain high-performance ethylene/ $\alpha$-olefin copolymers by designing suitable metallocene/methylaluminoxane (MAO)@POPs systems, there is a need to understand how the pore structure influences the molecular structure (e.g., molecular weight, molecular weight distribution, comonomer(s) incorporation, and chemical composition distribution-CCD), which are highly related to the physical performance of products made with an ethylene $/ \alpha$-olefin copolymer [33].

In thecurrent paper, ethylene/1-hexene copolymerization was conducted using a 2-hydroxyethylmethacrylate (HEMA)-functionalized POPs-supported ( $n$ - $\mathrm{BuCp})_{2} \mathrm{ZrCl}_{2} / \mathrm{MAO} /$ metallocene system. We investigated how the molecular chain length, molecular weight distribution, branching degree, and thechemical composition distributions of the produced ethylene/1-hexene copolymer were influenced by the active species scattered in the POPs-supported metallocene catalysts with well-defined pore structure.

\section{Results and Discussion}

\subsection{Pore Structure of POPs and POPs-Supported Metallocene Catalysts}

In this work, several POPs (POP-1/POP-2/POP-3) were preparedin 2-methyl-1-propanol, ethanol/deionized water (volume ratio $=9: 1$ ), and ethyl acetate, respectively, according to a dispersion polymerization method [30]. Then, the prepared POPs were used as metallocene catalyst supports to obtain POPs-supported metallocene catalysts.

The pore structure of the POPs and the POPs-supported metallocene catalysts were characterized with a Nova 2000e instrument; the porosity parameters results are shown in Table 1. In a previous paperwe showed [32] that the pore structure of the immobilized metallocene catalyst is highly dependent on the pore structure of the POPs with similar pore size distributions, except that a mild left shift of pore size and decrease of abundance of pore size are observed on the supported metallocene catalysts due to the non-covalent bonding of zirconocene complex/MAO to the POPs. From Table 1, we can see that the prepared POPs obtained different pore structures, with pore diameter ranging from $4 \mathrm{~nm}$ to $12 \mathrm{~nm}$, specific surface area (SSA) ranging from $300 \mathrm{~m}^{2} / \mathrm{g}$ to $450 \mathrm{~m}^{2} / \mathrm{g}$, and the supported metallocene catalysts also obtained similar pore structure with a mild decrease of average pore size ranging from $3 \mathrm{~nm}$ to $11 \mathrm{~nm}$, as mentioned above. However, compared with the POPs and POPs-supported metallocene catalysts, a dramatic decrease of average pore size of silicafrom $21.0 \mathrm{~nm}$ to $9.68 \mathrm{~nm}$ was observed when silica 2408 was used as the metallocene support. This shows that the prepared POP supports have better pore structure stability than inorganic silica as metallocene catalyst support. 
Table 1. Characterization data of $\mathrm{P}(\mathrm{HEMA}-\mathrm{co}-\mathrm{DVB})$ particles and $(n-\mathrm{BuCp})_{2} \mathrm{ZrCl}_{2} / \mathrm{MAO} / \mathrm{POPs}$ metallocene catalysts (BCZ/MAO@POPs) from $\mathrm{N}_{2}$ sorption results.BET: Brunauer-Emmett-Teller; HEMA: 2-hydroxyethylmethacrylate; MAO: methylaluminoxane; POP: porous organic polymer.

\begin{tabular}{|c|c|c|c|c|}
\hline Entry & Sample & $\begin{array}{l}\text { Specific Surface Area } \\
\text { (Multi Point BET) }\left(\mathrm{m}^{2} / \mathrm{g}\right)\end{array}$ & $\begin{array}{l}\text { Total Pore Volume } \\
\left(\mathrm{cm}^{3} / \mathrm{g}\right)\end{array}$ & $\begin{array}{c}\text { Average Pore } \\
\text { Diameter(nm) }\end{array}$ \\
\hline $1-1$ & POP-1 & 427 & 0.655 & 6.14 \\
\hline $1-2$ & BCZ/MAO@POP-1 & 299 & 0.318 & 4.26 \\
\hline $2-1$ & POP-2 & 414 & 0.416 & 4.02 \\
\hline $2-2$ & BCZ/MAO@POP-2 & 261 & 0.219 & 3.36 \\
\hline $3-1$ & POP-3 & 318 & 0.893 & 11.2 \\
\hline $3-2$ & BCZ/MAO@POP-3 & 288 & 0.730 & 10.1 \\
\hline $4-1$ & Silica-2408 & 279 & 1.47 & 21.0 \\
\hline $4-2$ & BCZ/MAO@Silica-2408 & 272 & 0.658 & 9.68 \\
\hline
\end{tabular}

The nitrogen sorption isotherms of the three POPs and silica 2408 are shown in Figure 1a. From the curves we can observe that very different porous structures were obtained. In order to investigate how the pore structure of the POPs influenced the chain growth of the ethylene/1-hexene copolymer, non-local density functional theory (NLDFT) simulation was adopted to determine the pore size distribution using $\mathrm{N}_{2}$-carbon kernel at $77 \mathrm{~K}$ based on a slit-pore model. As shown in Figure $1 \mathrm{~b}, \mathrm{c}$, the pore size and the pore size distribution of three POP supports (POP-1/POP-2/POP-3) varied greatly due to the different solvent parameters of the three types of solvents. However, despite the different pore size distribution, a similarity exists in the pore structure of the three POP supports, such that high abundance in the pore size of 1-4 nm scale can be observed in their NLDFT curves. Furthermore, the pore size distribution of the HEMA-functionalized POPs could be easily controlled by the selection of different HEMA/divinylbenzene (DVB) molar ratio or different solvent [29,30]. In contrast, the pore sizes of silica 2408 mainly focused in the meso-pore range (roughly 6-30 nm).
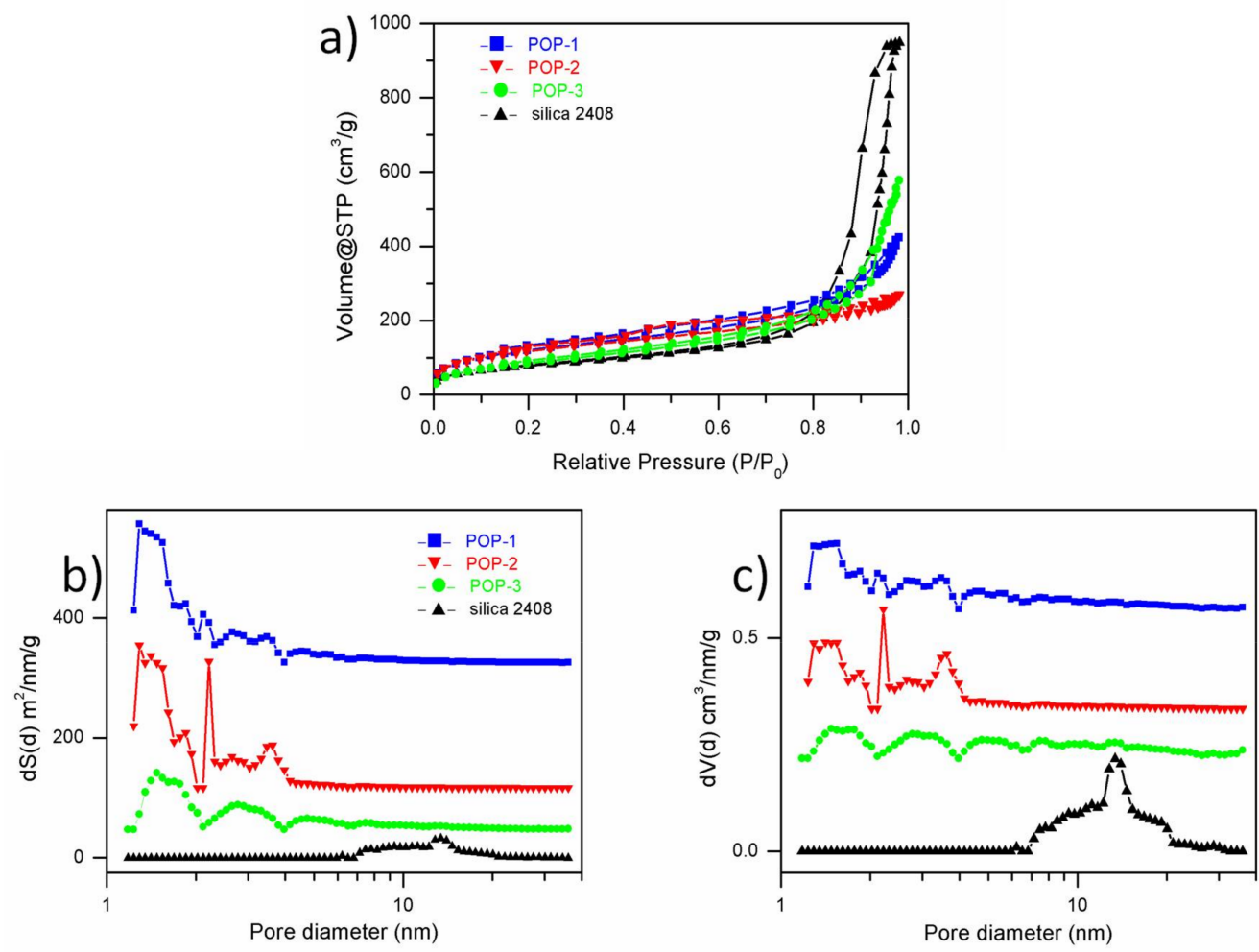

Figure 1. (a) $\mathrm{N}_{2}$ isotherms. Non-local density functional theory (NLDFT) pore size distribution curves of (b) dS vs. d and (c) dV vs. d of POPs and silica 2408. 


\subsection{Polymerization Activity and Active Sites on the POPs-Supported Metallocene Catalysts}

To evaluate the molecular structure of the ethylene/1-hexene copolymer, ethylene polymerizations were conducted using the POPs-supported $(n-\mathrm{BuCp})_{2} \mathrm{ZrCl}_{2} / \mathrm{MAO}$ catalysts in a slurry process. The supported metallocene catalysts displayed excellent $\alpha$-olefin incorporation ability and high ethylene/1-hexene copolymerization activity, and ethylene/1-hexene copolymerization results are shown in Table 2.

Surprisingly, sharply different loading amounts of zirconium and aluminum of the four supported metallocene catalysts from ICP (Inductive Coupled Plasma Emission Spectrometer) analysis were observed through the same $\left.(n-\mathrm{BuCp})_{2} \mathrm{ZrCl}_{2}\right] / \mathrm{MAO} /$ Supports immobilization formula. Thus, the same active species were obtained in the foursupported metallocene catalysts. From Table 2, we can see that the highest zirconium and aluminum contents $\left(\mathrm{Zr}=29.6 \mu \mathrm{mol} \mathrm{g}^{-1}, \mathrm{Al} / \mathrm{Zr}=172\right)$ were achieved from the silica gel-supported metallocene catalyst, which could be caused by strong interaction of MAO with the highly hydrophilic surface of the silica gel, and relatively low zirconium and aluminum content on the POPs-supported metallocene catalysts were obtained due to the less-hydrophilic surface of the poly(DVB-co-HEMA) porous polymers. Furthermore, even in the three POPs-supported catalysts, varied zirconium and aluminum contents were noticed with similar chemical component of the supports. This discrepancy might be caused by the different pore structure of the porous polymers-especially the specific surface area.

As for the ethylene/1-hexene polymerization results, the highest polymerization activity $(10,583 \mathrm{Kg}$ PE/molZr bar h) was not obtained from highest Zr loading catalyst, but from the catalyst (BCZ/MAO@POP-2) with the highest Al/Zr ratio. This proves that not all loading zirconium in the supported metallocene catalysts are effective active sites, and the $\mathrm{MAO} / \mathrm{Zr}$ ratio is another key factor in ethylene polymerization. In this regard, the BCZ/MAO@POP-2 catalyst is commercially beneficial for up-scaling.

\subsection{Chain Growth of Ethylene/1-Hexene Copolymer}

The $\alpha$-olefin comonomer effect was clearly observed during the process of ethylene/1-hexene copolymerization, as the copolymerization activities increased obviously in the presence of 1-hexene, compared with the ethylene homopolymerization results [32]. Gel permeation chromatographic (GPC) analysis revealed that the molecular weights of ethylene/1-hexene copolymer decreased from about 200,000 to 110,000 compared to ethylene homopolymer, and similar results were also reported for ethylene/ $\alpha$-olefin copolymers with other single-site catalyst systems [34,35]. The introduction of 1-hexene affects the catalytic activities and molecular chain length in different ways. Although the 1-hexene has stronger electron-donating ability than ethylene (which would benefit the coordination of 1-hexene to the active species and stabilizes the cationic active sites), the insertion rate of 1-hexene is slower than that of ethylene due to its steric hindrance [33]. The final results of the copolymerization showed that the increased activities and decreased molecular chain length might be caused by the increasing chain propagation rate and chain transfer rate combined, which could also be explained by the $\alpha$-olefin comonomer effect.

There is another important factor that can significantly influence the molecular chain length of polyolefin - the pore size structure. Generally, the average molecular weights of PE from polymer-supported catalysts are lower than that of inorganic-supported catalysts [36,37], because the porous polymer support is not as firm as mesoporous silica, and the soft polymer support will further result in quick chain termination. However, much higher average molecular weights of PE homopolymer were obtained from the POPs-supported metallocene catalysts, with pore size mainly scattering in the micro- and the narrow meso-pore $(1-4 \mathrm{~nm})$ range [32]. Similar results were also obtained, such that the average molecular weight of ethylene/1-hexene copolymers from the POPs-supported metallocene catalysts were higher than from silica-supported metallocene catalysts $\left(\mathrm{Mw}\right.$ : $\left.9.7 \times 10^{4}\right)$ when introducing 1-hexene in process of ethylene polymerization. 
The pore structure of the POP supports could be used to tune the molecular weight distribution or polydispersity, as the processability of polyolefin materials [38] was highly related to the molecular weight distribution of the prepared polyolefin. It is practical to improve the processability of metallocene-based polyolefin materials such as LLDPE (1-hexene or 1-octene as comonomer) by using multiple metallocene complexes or multinuclear metallocene catalysts and a multiple-reactor process to increase molecular weight distribution. We have reported the weight-average molecular weights $(\mathrm{Mw})$ of homo-polyethylene from the POPs-supported metallocene catalysts in the range of 90,000 to 400,000 , and their polydispersities of 2-3 are typically narrower than that of PE produced by silica-supported metallocene catalyst due to their ability to provide much closer analogue to the environment prevailing in homogeneous polymerization. By tuning the distribution of the active species scattered in the micro- and the narrow meso-pore $(1-4 \mathrm{~nm})$, the chain length of the polyolefin can be tailored effectively during the polymerization process. That is, the smaller the pore size of the POP support, the longer the chain length of PE that can be obtained due to the different diffusion rates of different sizes of monomer (ethylene), comonomer ( $\alpha$-olefin), hydrogen, and chain transfer agent (scavenger TIBA/TEAL), etc. Therefore, by tuning the pore size and pore size distribution of the POP supports, the molecular weight distribution of the prepared polyolefin can be tuned.

When an $\alpha$-olefin with larger size was added in the process of ethylene polymerization, the selectivity of diffusion of larger molecular $\alpha$-olefin to active species scattered in the in the microand the narrow meso-pore (1-4 nm) from POPs-supported metallocene catalysts would be better. As seen from Table 2, the molecular weight distribution of PE-4 from mesopore silica-supported metallocene was 2.9, and the molecular weight distribution of PE-1, PE-2, and PE-3 varied from 2.5 to 3.3 from different size and pore size distributions of POPs-supported metallocene catalysts. The molecular weight distribution of PE-1 from POP-1-supported metallocene catalyst was even higher than that of PE-4 from silica-supported metallocene catalyst, which proves that it is also a practical choice to improve the molecular weight distribution by tuning the pore size and pore size distribution of the POP supports.

A comparison of NLDFT pore size distribution curves (Figure 1) with GPC curves (Figure 2) offers some insight about this method of controlling molecular weight distribution. From Figure 1, we can see that the pore size and pore size distributions of POP-2 are mainly concentrated in microporous and narrow mesoporous range (roughly $1-4 \mathrm{~nm}$ ), while the pore size and pore size distributions of POP-1 are scattered in a relatively wide range (roughly $1-10 \mathrm{~nm}$ ). Because of the different diffusion rates of 1-hexene in different pore sizes, the active species scattered in the micro-pores and narrow meso-pores would make it more difficult to incorporate the 1-hexene molecule than in the larger meso-pores $(>4 \mathrm{~nm})$, thus resulting in longer molecular chain length from the active species scattered in the micro-pores and narrow meso-pores and shorter molecular chain length from the active species scattered in the larger meso-pores. The part of the PE-1molecular chain with shorter chain length was observed in GPC curves compared with PE-2, while the part of PE-1molecular chain with higher chain length was almost overlapping with that of PE-2, or slightly higher that of PE-2 in the highest molecular weight area. Therefore, the wide molecular weight distribution of PE- 1 was obtained from the POP-1-supported metallocene catalyst with wide pore size distribution. 
Table 2. Ethylene/1-hexene polymerization activities, differential scanning calorimetry (DSC) and gel permeation chromatography (GPC) results of polyethylene (PE) from the POPs-supported $n$ - $\mathrm{BuCp}_{2} \mathrm{ZrCl}_{2} / \mathrm{MAO}$ metallocene catalysts. PDI: polydispersity index.

\begin{tabular}{|c|c|c|c|c|c|c|c|c|c|c|c|c|c|}
\hline \multirow[b]{2}{*}{ Entry } & \multirow[b]{2}{*}{ Catalyst } & \multicolumn{2}{|c|}{ Catalyst Component } & \multirow{2}{*}{$\begin{array}{c}\text { Polymerization } \\
\text { Activity } \\
\text { (Kg PE/molZr bar h) }\end{array}$} & \multicolumn{2}{|c|}{ DSC Results } & \multirow[b]{2}{*}{$\underset{\mathrm{J} / \mathrm{g}}{\Delta \mathrm{Hm}}$} & \multicolumn{3}{|c|}{ GPC Results } & \multicolumn{3}{|c|}{ IR Results } \\
\hline & & $\underset{(\mu \mathrm{mol} \mathrm{g}}{\mathrm{Zr}}$ & $\underset{(\mathrm{mol} / \mathrm{mol})}{\mathrm{Al} / \mathrm{m}}$ & & $\begin{array}{c}\mathrm{Tm} \text { (Peak) } \\
{ }^{\circ} \mathrm{C}\end{array}$ & $\begin{array}{c}\mathrm{Tc} \text { (Peak) } \\
{ }^{\circ} \mathrm{C}\end{array}$ & & $\begin{array}{c}\mathrm{Mw} \\
\left(\times 10^{4}\right)\end{array}$ & $\underset{\left(\times 10^{4}\right)}{\mathrm{Mn}}$ & PDI & $\begin{array}{l}\text { Branching } \\
\text { Degree } \\
(/ 1000 \mathrm{C})\end{array}$ & $\begin{array}{c}\mathrm{RCH}=\mathrm{CH}_{2} \\
\text { Relative } \\
\text { Content }\end{array}$ & $\begin{array}{c}\mathrm{R}_{1} \mathrm{R}_{2} \mathrm{C}=\mathrm{CH}_{2} \\
\text { Relative } \\
\text { Content }\end{array}$ \\
\hline PE-1 & BCZ/MAO@ POP-1 & 27.4 & 178 & 7105 & 120.7 & 108.2 & 123.8 & 11.2 & 3.4 & 3.3 & 10.1 & 0.05 & $0.11-0.12$ \\
\hline PE-2 & BCZ/MAO@ POP-2 & 14.3 & 280 & 10,583 & 121.7 & 103.8 & 131.1 & 11.2 & 4.4 & 2.5 & $10.9-11.6$ & $0.04-0.06$ & $0.10-0.14$ \\
\hline PE-3 & BCZ/MAO@ POP-3 & 18.6 & 163 & 4075 & 121.7 & 107.4 & 127.1 & 13.5 & 5.2 & 2.6 & $10.7-11.2$ & $0.02-0.04$ & $0.05-0.10$ \\
\hline PE-4 & BCZ/MAO@Silica-2408 & 29.6 & 172 & 7387 & 120.0 & 108.1 & 120.6 & 9.7 & 3.3 & 2.9 & 11.4 & 0.04 & 0.09 \\
\hline
\end{tabular}




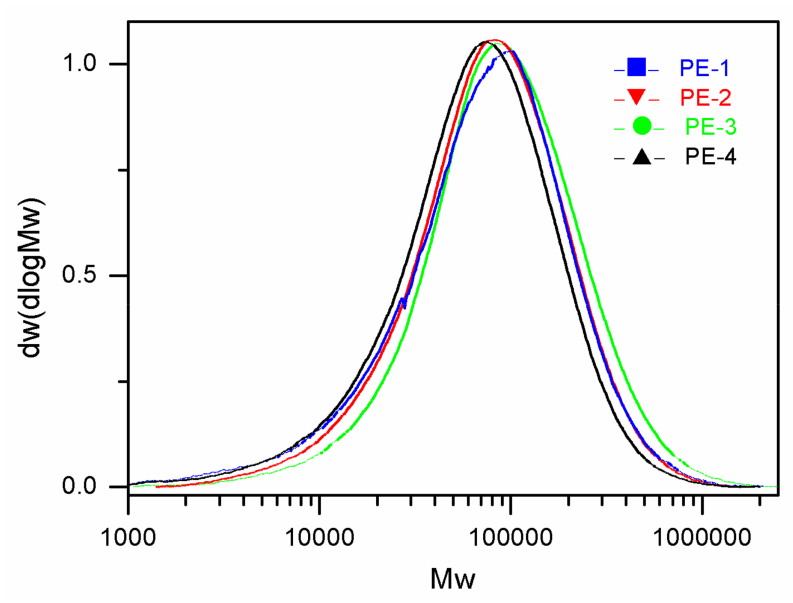

Figure 2. Curves of the molecular weight distribution of ethylene/1-hexene copolymers. (1) PE-1 obtained from BCZ/MAO@POP-1metallocene catalyst; (2) PE-2 obtained using BCZ/MAO@POP-2metallocene catalyst; (3) PE-3 obtained from BCZ/MAO@POP-6 metallocenecatalyst; (4) PE-4 obtained from BCZ/MAO@2408 silicametallocene catalyst.

However, compared to PE-1, the molecular weight distribution of PE-3 (Mw/Mn = 2.6) from the POP-3-supported metallocene catalyst with wide pore size distribution seemed to be narrower than PE-1. The results could be explained reasonably by the same diffusion mechanism in different pore sizes. Besides the pore size distribution, the abundance in different pore size distributions might cause the discrepancy in wide pore size distribution POP supports. Actually, the abundance in $1-4 \mathrm{~nm}$ of POP-3 is the highest in the three POP supports, so the part of PE-3 with high molecular weight is also the highest in the three copolymers (PE-1/PE-2/P-3), and many more long-chain molecules with $\mathrm{Mw}$ above $20 \times 10^{4}$ are in PE-3 than in PE- 1 and PE-2. On the other hand, many more short chain molecules with Mw less than 2000 in PE-3 were observed than in PE-2, which might owe to the large pores in the POP-3. Furthermore, compared to PE-4 obtained from meso-pore silica-supported metallocene catalyst, an obvious shift to the right of the GPC curves of PE-1/PE-2/PE-3 due to the micro-pores and narrow meso-pores (roughly 1-4 nm) in the POP supports was seen, except for the part of low molecular weight, with Mw less than about 2000.

The incorporation mechanism of ethylene and 1-hexene by diffusion through different pore sizes of the POPs-supported metallocene catalysts is illustrated in Figure 3. In this case, the nanoporous POP supports or POPs-supported metallocene catalysts-especially in microporous and narrow mesoporous range-would behave like nano-valves which can differently affect the diffusion rate of monomer (ethylene), comonomer ( $\alpha$-olefin), hydrogen, and chain transfer agent (scavenger TIBA/TEAL), etc., according to their molecule size, while larger meso-pores cannot effectively hold back the diffusion of larger molecules such as 1-hexene and TIBA.

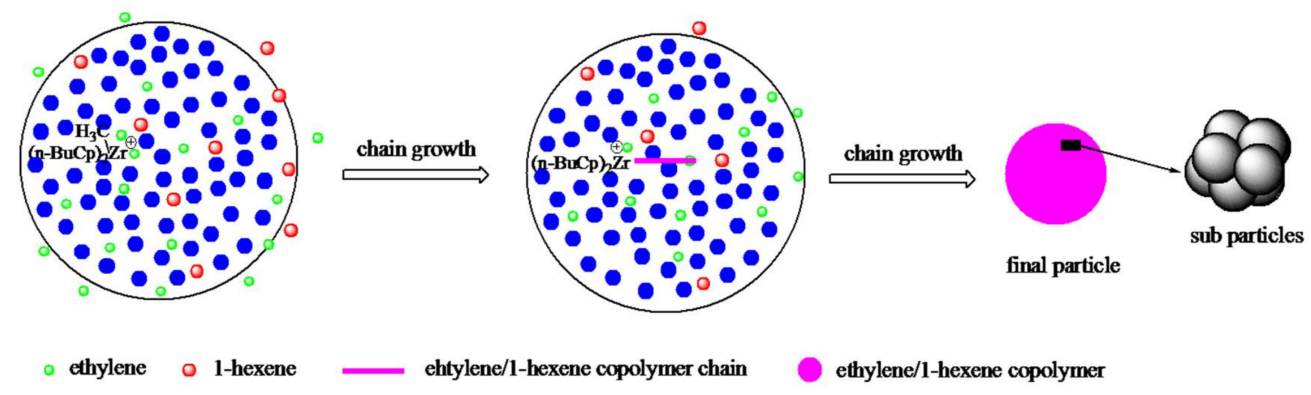

Figure 3. Schematic incorporation mechanism of comonomers by diffusion through different pore sizes of the POPs-supported metallocene catalysts during ethylene/1-hexene copolymerization. 


\subsection{Molecular Structure Analysis from IR Results}

The microstructure of PE could be characterized by IR spectroscopy. The branching degree and relative double bond content of polyethylene can be calculated by IR analysis results [39-41]. Figure 4 shows the spectra of PE-1, PE-2, and PE-3 at room temperature. The $1378 \mathrm{~cm}^{-1}$ peak is the characteristic peak of the methyl group due to its deformation vibration, and the absorbance in the methyl peak could be used to determine the branching degree. The $1369 \mathrm{~cm}^{-1}$ peak and $1352 \mathrm{~cm}^{-1}$ peak are caused by conformational $\mathrm{CH}_{2}$ wagging, which might overlap the methyl absorbance at $1378 \mathrm{~cm}^{-1}$, but they do not cause significant interference in this test method because their intensities are not significantly affected by the comonomer content, but rather by the plaque thickness. The band at $2019 \mathrm{~cm}^{-1}$ was used as internal thickness correction approach to calibrate the methyl content. The branching degree of PE can be calculated by determining the value of absorbance (A) at $1378 \mathrm{~cm}^{-1} /$ area $\left(2019 \mathrm{~cm}^{-1}\right)$ in a standard curve, which was plotted in $\mathrm{A}\left(1378 \mathrm{~cm}^{-1}\right) /$ Area $\left(2019 \mathrm{~cm}^{-1}\right)$ vs. Number of branches $(\mathrm{N})$ per 1000 carbons as determined by ${ }^{13} \mathrm{C}$ NMR results.

The peaks at $908 \mathrm{~cm}^{-1}$ and $888 \mathrm{~cm}^{-1}$ are the characteristic peaks of the unsaturated groups of PE. The peak at $908 \mathrm{~cm}^{-1}$ is caused by the wagging vibration of $\mathrm{CH}_{2}$ in $\mathrm{RCH}=\mathrm{CH}_{2}$ groups, and the peak at $888 \mathrm{~cm}^{-1}$ is caused by the same vibration mode of $\mathrm{CH}_{2}$ in $\mathrm{R}_{1} \mathrm{R}_{2} \mathrm{C}=\mathrm{CH}_{2}$ groups. According to the similar internal standard to correct the variation, the relative content of $\mathrm{RCH}=\mathrm{CH}_{2}$ groups and $\mathrm{R}_{1} \mathrm{R}_{2} \mathrm{C}=\mathrm{CH}_{2}$ groups could be obtained by Area $\left(908 \mathrm{~cm}^{-1}\right) /$ Area $\left(2019 \mathrm{~cm}^{-1}\right)$ and Area $\left(888 \mathrm{~cm}^{-1}\right) /$ Area $\left(2019 \mathrm{~cm}^{-1}\right)$, respectively.

The results of branching degree as well as relative content of $\mathrm{RCH}=\mathrm{CH}_{2}$ groups and $\mathrm{R}_{1} \mathrm{R}_{2} \mathrm{C}=\mathrm{CH}_{2}$ groups are listed in Table 2 in accordance with the method above. From Table 2, we can see that the branching degree of these ethylene/1-hexene copolymers varied from 10.1 to 11.6 , and the branching degree of PE-4 (11.4) was nearly the highest among them, despite their different polymerization activity. This variation can be partly explained by the controlling diffusion of ethylene and $\alpha$-olefin, etc. to the active sites by the nanoporous POP supports with narrower pore size than silica; in turn, the different concentrations of ethylene and $\alpha$-olefin determine different chain growth rates and chain transfer rates of the active sites. However, the results of PE branching degree seemed to have no obvious bearing on the relative content of unsaturated groups due to their different formation mechanism [42], as seen from Table 2. The formation of $\mathrm{RCH}=\mathrm{CH}_{2}$ groups and the $\mathrm{R}_{1} \mathrm{R}_{2} \mathrm{C}=\mathrm{CH}_{2}$ groups are caused by $\beta$-hydrogen elimination to $\mathrm{Zr}+$ and/or to ethylene or the comonomer, while the branching degree of $\mathrm{PE}$ is mainly generated by the insertion of 1-hexene. PE-1 had the lowest branching degree of 10.1 , while the relative content of $\mathrm{RCH}=\mathrm{CH}_{2}$ groups and $\mathrm{R}_{1} \mathrm{R}_{2} \mathrm{C}=\mathrm{CH}_{2}$ groups were 0.05 and $0.11-0.12$, respectively. Moreover, the addition of 1-hexene caused a dramatic increase of $\mathrm{R}_{1} \mathrm{R}_{2} \mathrm{C}=\mathrm{CH}_{2}$ groups of ethylene/1-hexene copolymer compared to ethylene homopolymer (0.01-0.04). 

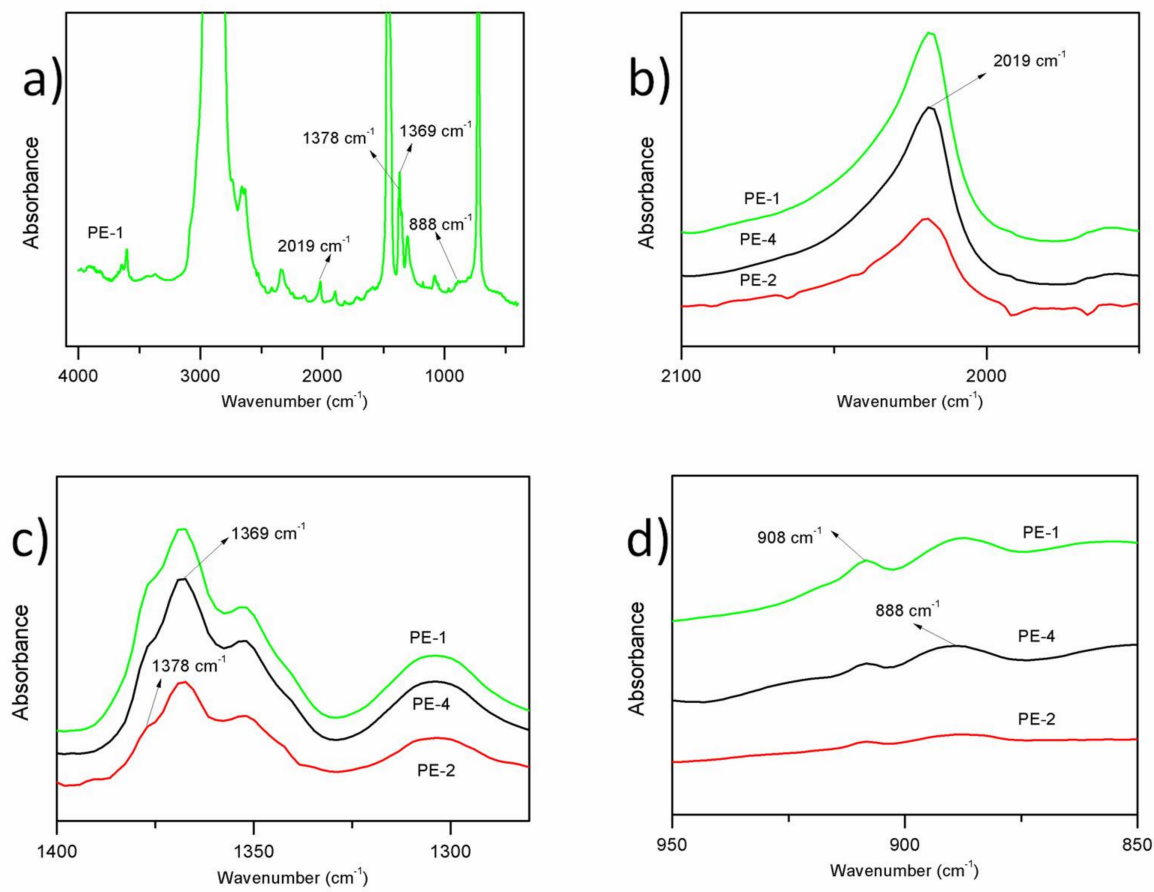

Figure 4. IR analysis results. (a) IR spectrum of PE-1; (b) IR spectrum at $2019 \mathrm{~cm}^{-1}$; (c) IR spectrum of methyl groups at $1378 \mathrm{~cm}^{-1}$ and methylene groups at $1369 \mathrm{~cm}^{-1}$; (d) IR spectrum of $\mathrm{RCH}=\mathrm{CH}_{2}$ groups at $908 \mathrm{~cm}^{-1}$ and $\mathrm{R}_{1} \mathrm{R}_{2} \mathrm{C}=\mathrm{CH}_{2}$ groups at $888 \mathrm{~cm}^{-1}$.

\subsection{Differential Scannning Calorimetry (DSC) and Temperature Rising Elution Fractionation (TREF) Results}

The TREF technique was adopted to characterize the chemical composition distributions (CCDs) of the produced ethylene/1-hexene copolymers prepared from POPs- and silica-supported metallocene catalysts. Figure 5a shows the TREF analysis of PE copolymers from POPs- and silica-supported metallocene catalysts. Although similar TREF curves in these PE samples are observed, the specific results of soluble fraction and fractionation temperature peaks are different. The soluble fractions at room temperature of ethylene/1-hexene copolymers prepared from POPs-supported metallocene catalysts-which mainly contain very-low-molecular-weight PE molecules ( $\mathrm{Mw}<1000 \mathrm{~g} / \mathrm{mol}$ ) [43]—were higher than from silica-supported metallocene catalyst. An interesting phenomenon is also observed from Table 3-the ethylene/1-hexene copolymers (PE-1/PE-2/PE-3) prepared from POPs-supported metallocene catalysts have relatively less fraction in the main elution peak around $94{ }^{\circ} \mathrm{C}$ (due to less relative content of branching, especially short chain branching-SCB) and have a greater fraction in the secondary elution peak around $52-64{ }^{\circ} \mathrm{C}$ (due to a greater relative content of SCB) than PE-4 from silica-supported metallocene catalyst. From this evidence, we can reasonably conclude that the active sites in the nanoporous POP supports would facilitate redistributions of SCB in the main peak and the secondary peak by tuning the diffusion of ethylene and 1-hexene, etc. A wider distribution of pore size of POP-1 and POP-3 obviously had a better effect on the redistributions of SCB with PE-1 and PE-3.PE-1 had a soluble fraction of $1.8 \%, 96.3 \%$ around the main peak at $94{ }^{\circ} \mathrm{C}$ and $0.7 \%$ around the secondary peak at $52{ }^{\circ} \mathrm{C}$, and PE-3 had a soluble fraction of $0.6 \%, 96.8 \%$ around the main peak at $94^{\circ} \mathrm{C}$ and $1.0 \%$ around the secondary peak at $52{ }^{\circ} \mathrm{C}$. 
Table 3. Temperature rising elution fractionation (TREF) analysis results.

\begin{tabular}{cccccc}
\hline Entry & Support & & Soluble Fraction (SF) & Peak 1 & Peak 2 \\
\hline PE-1 & POP-1 & $\mathrm{T} /{ }^{\circ} \mathrm{C}$ & & 52.6 & 94.2 \\
& & Area $/ \%$ & 1.8 & 0.7 & 96.3 \\
PE-2 & POP-2 & $\mathrm{T} /{ }^{\circ} \mathrm{C}$ & & 63.7 & 94.5 \\
& & Area $/ \%$ & 0.3 & 0.5 & 98.6 \\
PE-3 & POP-3 & $\mathrm{T} /{ }^{\circ} \mathrm{C}$ & & 61.8 & 94.7 \\
& & Area $/ \%$ & 0.6 & 1.0 & 96.8 \\
PE-4 & Sylopol 2408 & $\mathrm{~T} /{ }^{\circ} \mathrm{C}$ & & 53.1 & 94.2 \\
& & Area $/ \%$ & 0.2 & 0.4 & 98.9 \\
\hline
\end{tabular}

Differential scanning calorimetry (DSC) results (Figure 5b) reveal that the ethylene/1-hexene copolymers from both POPs- and silica-supported metallocene catalysts had similar thermal behavior with $\mathrm{Tm}$ (peak) in the range of $120-122^{\circ} \mathrm{C}$ and $\mathrm{Tc}$ (peak) in the range of $103-109^{\circ} \mathrm{C}$, which was mainly caused by the same active site species in the polymerization process. As for crystalline polymer, the crystallinity $\mathrm{Xc}_{\mathrm{c}}$ of polymer can be estimated by the following equation:

$$
\mathrm{X}_{\mathrm{c}}=\Delta \mathrm{Hm} / \Delta \mathrm{H}^{0} \mathrm{~m},
$$

where $\Delta \mathrm{Hm}$ is the melting endothermic enthalpy of the polymer, and $\Delta \mathrm{H}^{0} \mathrm{~m}$ is the melting endothermic enthalpy of the polymer with $100 \%$ crystallinity.

According to this Equation (1), the values of $\Delta \mathrm{Hm}$ of ethylene/1-hexene copolymers could be estimated for their crystallinity. From Table 2, we can see the $\Delta \mathrm{Hm}$ of PE-4 had the lowest value of $120.6 \mathrm{~J} / \mathrm{g}$. Therefore, the lowest crystallinity of PE-4 among these samples could be estimated, which is consistent with nearly the highest branching degree from the IR results. Although a discrepancy between the values of $\Delta \mathrm{Hm}$ from PE-1/PE-2/PE-3 and their branching degree was observed, the discrepancy combined with a relatively wide range of Tc (peak) value of these PE samples might be partly explained by the different distribution and content of SCB in the their chains, which was caused by tuning the diffusion of ethylene and 1-hexene in different pore sizes. Furthermore, a minor peak with $\Delta \mathrm{Hc}$ of about $4.3 \mathrm{~J} / \mathrm{g}$ on the crystalline curves of PE- 1 or PE- 4 around $73{ }^{\circ} \mathrm{C}$ was observed as seen from Figure $5 b$, and this small crystalline peak is in agreement with the TREF signals of the secondary peak of these PE samples-both of which are caused by the crystalline molecules with relatively high SCB content.
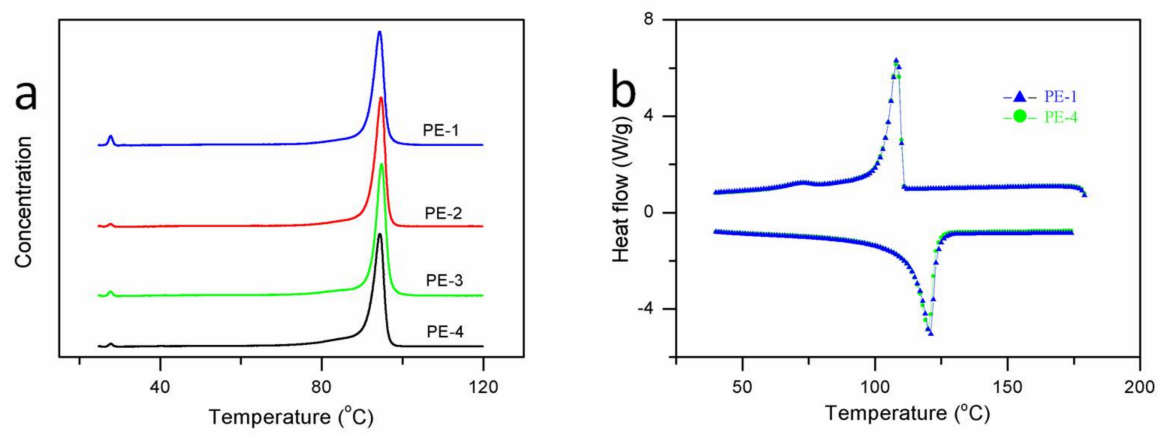

Figure 5. (a) TREF analysis of PE copolymers produced from POPs- and silica-supported metallocene catalysts; (b) DSC analysis of PE-1 and PE-4.

\subsection{Surface Morphology}

The morphology of the PEwas characterized by scanning electron microscopy (SEM). The SEM image in Figure 6 clearly shows that the ethylene/1-hexene copolymers have different morphology due to the different pore structure of their catalyst supports. PE-1 hada highly porous fabric consistingof 
fiber and spherical beads of micron dimension, PE-2 had porous structure aggregated mainly by spherical beads, and PE-4 was aggregated by spherical beads with fila around the beads. The different morphologies of these PE samples can be explained reasonably by the replicating effect of the PE product on the porous POP and silica in the olefin polymerization process [36,37].The porous support played as a template on two levels [36], taking the overall morphology of product polymer into account. One level is the whole support beads, which resulted in the porous polyethylene particles. Another level is the porous structure in the support beads, which played as nanoreactors and resulted in the fiber structure in the polyethylene particles. According to this particle growth mechanism, the morphology of PE would be related to the polymerization time and activity, besides the porous structure of the support and the morphology of the support itself. With the continuous chain growth, the catalyst beads gradually became larger because of the expansion in the catalyst beads and external growth by the active sites in the outer surface; the morphology of PE-1 would be more like that of PE-2 if polymerization continued.
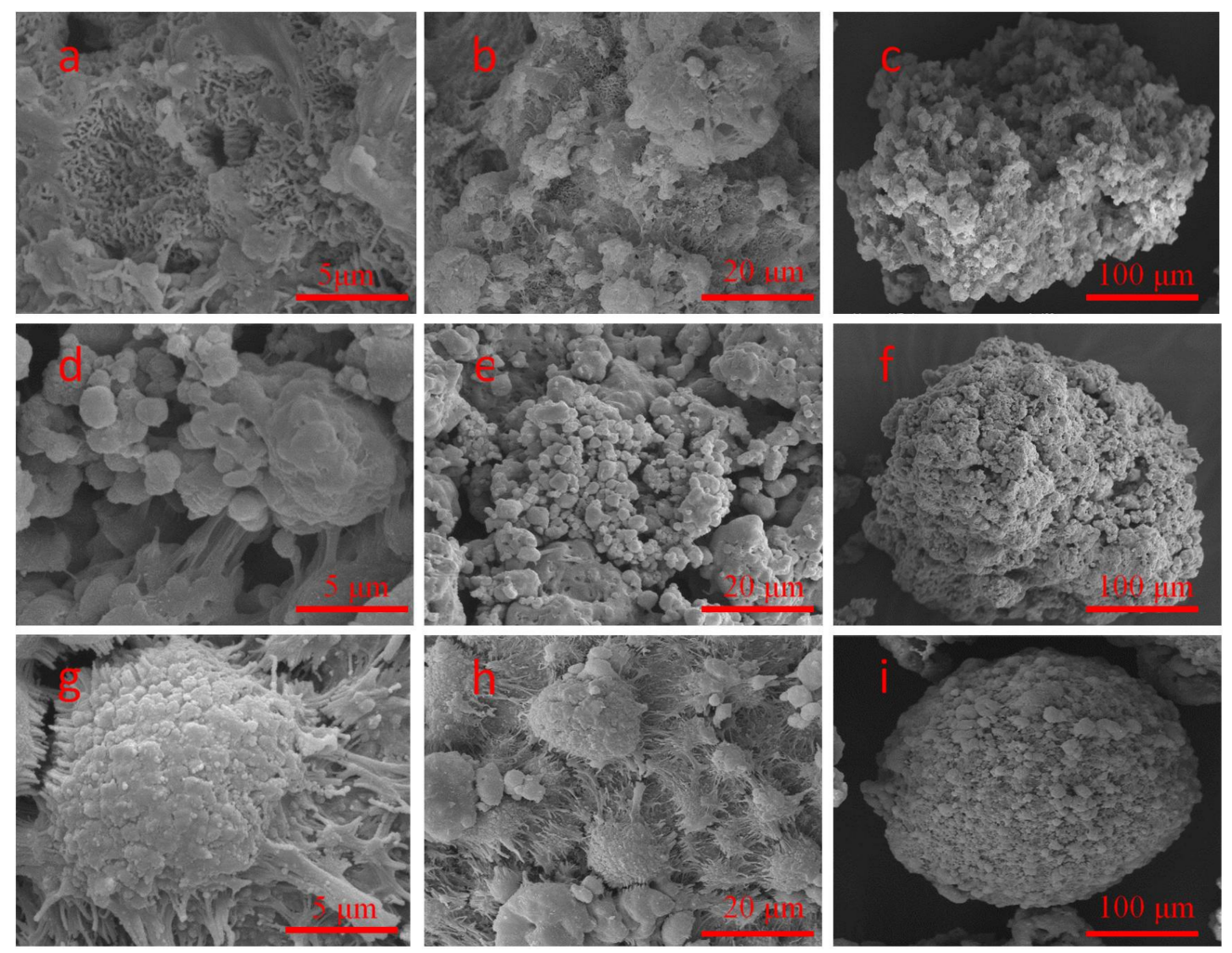

Figure 6. Scanning electron micrographs of PE copolymers particles. (a-c) PE-1 from POP-1-supported metallocene catalyst; (d-f) PE-2 from POP-2-supported metallocene catalyst; (g-i) PE-4 from 2408 silica-supported metallocene catalyst.

\subsection{Theoretical Explanation}

The distribution of chain length and chemical composition for ethylene/1-hexene copolymers can be interpreted based on the Stockmayer bivariate distribution [44]. The Stockmayer distribution is given by the following Equation (2):

$$
w_{r, F_{A}}=r \tau^{2} e^{-r \tau} \sqrt{\frac{r}{2 \pi \beta}} e^{-\frac{r\left(F_{A}-\overline{F_{A}}\right)^{2}}{2 \beta}}
$$

where the parameters $r$ and $\tau$ have the same meanings as in the Flory distribution; $F_{A}$ represents the molar fraction of monomer $\mathrm{A}$ in a copolymer chain; $\overline{\mathrm{F}_{\mathrm{A}}}$ is the average molar fraction of monomer $\mathrm{A}$ in 
the whole copolymer, and its value can also be measured by ${ }^{13} \mathrm{C}$ NMR and Fourier transform infrared spectroscopy (FTIR).The parameter $\beta$ is described as following Equation (3):

$$
\beta=\overline{F_{A}}\left(1-\overline{F_{A}}\right) \sqrt{1-4 \overline{F_{A}}\left(1-\overline{F_{A}}\right)\left(1-r_{A} r_{B}\right)} .
$$

By integrating Equation (2), the CCD component of the Stockmayer distribution can be changed into Equation (4):

$$
w_{F_{A}}=\frac{3 \tau^{2} \beta^{2}}{\left[2 \beta \tau+(F-\bar{F})^{2}\right]^{5 / 2}}=\frac{3}{4 \sqrt{2 \beta \tau}\left[1+\frac{(F-\bar{F})^{2}}{2 \beta \tau}\right]^{5 / 2}} .
$$

From Equation (4), we can infer that the CCD becomes broader as the product $\beta \tau$ increases, as expected that $\tau$ increases when $r_{n}$ decreases and $\beta$ increases when $r_{A} r_{B}$ increases, both factors broaden the CCD.

Soares and Mckenna [44] illustrated how the Stockmayer distribution was used to describe the CCD of ethylene/1-hexene copolymers prepared from a metallocene catalyst. The CRYSTAF peaks moved to lower crystallization temperatures and the profiles became broader as 1-hexene content increased. Stockmayer's distribution can be used successfully to explain this behavior. The Stockmayer parameters $\tau$ and $\beta$ were calculated, assuming that the copolymers are random $\left(\mathrm{r}_{\mathrm{A}} \mathrm{r}_{\mathrm{B}} \approx 1\right)$. The product $\beta \tau$ increases with increasing 1-hexene content in the polymer, so the CRYSTAF peaks were expected to become broader. As shown in Figure 7, that results are in good agreement with the experimental data. From Equation (4), we can infer that the CCD becomes broader as the product $\beta \tau$ increases. As expected from our previous analysis ofthe Stockmayer distribution, $\tau$ increases when $r_{n}$ decreases and $\beta$ increases when $r_{A} r_{B}$ increases, and both factors broaden the CCD.
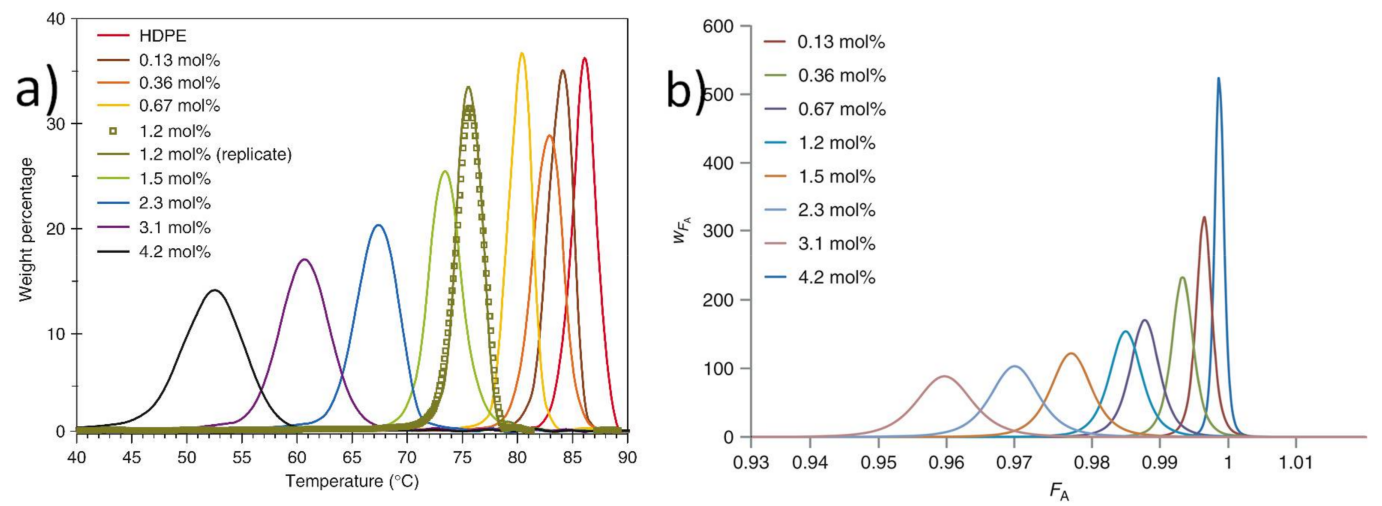

Figure 7. (a) CRYSTAF profiles of ethylene/1-hexene copolymers prepared from a single-site catalyst; (b) chemical composition distributions predicted with Stockmayer distribution [44]. CRYSTAF: is a fully automated instrument intended for the fast measurement of the Chemical Composition Distribution (CCD) in Polyolefins, and performs the Crystallization Analysis Fractionation technique to separate the polymer by its comonomer content. HDPE: high density polyethylene.

As in this case, through different diffusion rates of ethylene/ $\alpha$-olefin and TIBA to the active sites controlled by the different pore structure (especially the micro- and the narrow meso-pores) of the POP supports, different reaction micro-environments of the same active species were established in the nanoreactors, which caused the variations of concentration of 1-hexene and of $\beta \tau$ value, thus leading to the broadening of CCDs as seen from the TREF results in Table 3. Additionally, different CCDs and polydispersity indexes (PDIs) of these PE samples were observed from POP supports with different pore structure. From this perspective, the design and synthesis of well-defined POP-supported pore structure would be practical for controlling the end-use properties (e.g., the CCD and the molecular weight distribution) of the polyolefin. 


\section{Materials and Methods}

\subsection{Materials}

Divinylbenzene (DVB, 80\%, Aladdin Reagent, Shanghai, China) was treated with $\mathrm{NaOH}$ solution (5 wt \%) and washed with deionized water before use. 2-Hydroxyethylmethacrylate (HEMA) ( $\geq 98 \%$, Aladdin Reagent, Shanghai, China) was extracted with neutral oxide aluminum (200-300 mesh) column to remove the inhibitor and 2,2'-azo-bis-isobutyronitrile (AIBN) was recrystallized from methanol before use. Poly (vinyl alcohol) (PVA, 1788, 88\%) (Aladdin Reagent, Shanghai, China), ethanol ( $\geq 99.7 \%)$ (Aladdin Reagent, Shanghai, China), ethyl acetate $(\geq 99.8 \%)$ (Aladdin Reagent, Shanghai, China), 2-methyl-1-propanol ( $\geq 99.5 \%$, Aladdin Reagent, Shanghai, China), and deionized water (Lanzhou Petrochemical Research Center, Lanzhou, China) were used as received. 1-Hexene ( $\geq 99 \%$, TCI, Shanghai, China) was dried using 4 A molecular sieve and distilled before use. Bis( $n$-butylcyclopentadienyl) zirconium dichloride ( $n$-BuCp) ${ }_{2} \mathrm{ZrCl}_{2}$ (98\%, DALCHEM, Nizhny Novgorod, RU) and methylaluminoxane (MAO) (10 mass \% in toluene, PetroChina, Lanzhou, China) were used without further treatment.

\subsection{Synthesis of $P(H E M A-c o-D V B)$ POP Supports}

HEMA-functionalized porous organic polymers particles were synthesized according to a dispersion polymerization method reported by our group [29,30]. Briefly, $140 \mathrm{~mL}$ solvent was charged into a multi-necked glass reactor, then $0.0369 \mathrm{~mol}$ of DVB (80\%), 0.0246 mol of HEMA, and $2 \mathrm{wt} \%$ of PVA were added into the reactor when stirring. After the comonomers and the stabilizer were dissolved in the solution at $50{ }^{\circ} \mathrm{C}, 2 \mathrm{wt} \%$ of the initiator AIBN was added into the reactor to initiate the free radical polymerization at $70{ }^{\circ} \mathrm{C}$ for $3 \mathrm{~h}$. After aging for about $5 \mathrm{~h}$, the product was purified with solvent to remove impurities and vacuum filtered for further use. POP- 1 particles were prepared in 2-methyl-1-propanol, POP-2 particles were synthesized in deionized water/ethanol mixture (1:9 volume ratio), and POP-3 particles were synthesized with DVB/HEMA (2:1 molar ratio) in ethyl acetate.

\subsection{Supporting of Metallocene Catalyst}

The $(n-\mathrm{BuCp})_{2} \mathrm{ZrCl}_{2} / \mathrm{MAO}$ metallocene system was immobilized on the prepared POPs after they were vacuum-dried at $120{ }^{\circ} \mathrm{C}$ for $8 \mathrm{~h}$. Sylopol 2408 silica support was activated at $600{ }^{\circ} \mathrm{C}$ for $10 \mathrm{~h}$ at nitrogen atmosphere and used as control sample. The supporting procedure was with Schlenk and glove-box techniques under high-purity nitrogen according to previous work [29]. The supporting procedure is summarized as follows.

In a $250 \mathrm{~mL}$ multi-neck reactor, $2.4 \mathrm{~g}$ treated POP particles and $50 \mathrm{~mL}$ toluene were added, then $15 \mathrm{~mL}$ MAO (10 wt \%, in toluene) was added to the above suspension and stirred at room temperature for $1 \mathrm{~h}$. Then, $0.247 \mathrm{mmol}(n-\mathrm{BuCp})_{2} \mathrm{ZrCl}_{2}$ was added to the POP/MAO suspension and the obtained $(n-\mathrm{BuCp})_{2} \mathrm{ZrCl}_{2} / \mathrm{MAO} / \mathrm{POP}$ suspension was kept stirring for $3 \mathrm{~h}$ at $-20{ }^{\circ} \mathrm{C}$. Then, the solid was washed in toluene and hexane to remove impurities, and was vacuum dried to obtain the final catalyst particles.

\subsection{Ethylene/1-Hexene Polymerization Procedure}

Ethylene/1-hexene copolymerization was conducted in an $800 \mathrm{~mL}$ stainless steel reactor equipped with a stainless blade stirrer and an external oil jacket for temperature control; $350 \mathrm{~mL}$ hexane, $2 \mathrm{~mL}$ TEAL (1 M in hexane), and $3.5 \mathrm{~mL}$ 1-hexene were added successively into the reactor, then $140 \mathrm{mg}$ of the supported $(n \text {-BuCp })_{2} \mathrm{ZrCl}_{2} / \mathrm{MAO} @ P O P s$ catalysts were added under high-purity nitrogen. The nitrogen was then pumped out before introducing the ethylene. Temperature and pressure were then progressively increased up to $80^{\circ} \mathrm{C}$ and 3 bar. The pressure was kept constant during the polymerization. After $30 \mathrm{~min}$ of reaction, the polymerization was stopped. The polymer was collected by filtrationand dried. 
Ethylene/1-hexene copolymerization was conducted in an $800 \mathrm{~mL}$ pressure reactor equipped with a stainless blade stirrer and an external oil jacket for temperature control. $350 \mathrm{~mL}$ hexane, $2 \mathrm{~mL}$ TIBA (1 $\mathrm{M}$ in hexane), and $3.5 \mathrm{~mL}$ 1-hexene were added successively into the reactor, then $140 \mathrm{mg}$ of the supported ( $n$-BuCp) ${ }_{2} \mathrm{ZrCl}_{2} / \mathrm{MAO} @ P O P s$ catalysts were added under high-purity nitrogen. After the nitrogen was replaced with ethylene, the reactor temperature was increased up to $80^{\circ} \mathrm{C}$. Then, the reactor pressure was kept constant at 3 bar by introducing ethylene during the polymerization. After 30 min of reaction, the polymerization was terminated. The polymer was filtered and dried for analysis and characterization.

\subsection{Characterization}

Nitrogen sorption porosimetry was carried out on a Nova 2000e (Quantachrome Instruments, Boynton Beach, FL, USA). The POP samples were vacuum dried in a test tube at $120{ }^{\circ} \mathrm{C}$ for $8 \mathrm{~h}$ to remove adsorbed materials from the surface before $\mathrm{N}_{2}$ sorption $(77.3 \mathrm{~K})$. The supported metallocene catalysts were directly tested after sampling under nitrogen protection. $\mathrm{Al}$ and $\mathrm{Zr}$ contents of the supported metallocene catalysts were analyzed on a VISTA ICP-MPX (VARIAN, Palo Alto, CA, USA). Into a $10 \mathrm{~mL}$ mixture of $\mathrm{HClO}_{4} / \mathrm{HNO}_{3}: \mathrm{HCl}$ (1:3), $0.1 \mathrm{~g}$ catalyst was added, was dissolved by heating, then the solution was metered to $100 \mathrm{~mL}$ constant volume using $2 \% \mathrm{HCl}$ solution. Aluminum and zirconium contents were obtained from their characteristic peaks calibrated by their standard curves. Gel permeation chromatography (GPC) was conducted in a PL-GPC 220 instrument (Church Stretton, UK) using 1,2-dichlorobenzene as solvent at $135^{\circ} \mathrm{C}$ with a PE sampling concentration of $3 \mathrm{mg} / \mathrm{mL}$ and a sampling rate of $1.0 \mathrm{~mL} / \mathrm{min}$. IR analysis was performed on a NEXUS 670 FTIR (Glendale, WI, USA). Differential scanning calorimetry (DSC) measurements were analyzed on a DSC Q2000 (New Castle, DE, USA). To eliminate the heat history of test samples, $10 \mathrm{mg}$ PE was heated first from room temperature to $180{ }^{\circ} \mathrm{C}$ under nitrogen atmosphere at a heating rate of $10{ }^{\circ} \mathrm{C} / \mathrm{min}$, then cooled to room temperature at a cooling rate of $10{ }^{\circ} \mathrm{C} / \mathrm{min}$. The melting curves were obtained when heating the PE samples from room temperature to $180^{\circ} \mathrm{C}$ at the same heating rate once again, and crystallization curves were obtained when cooling the PE samples to $40{ }^{\circ} \mathrm{C}$. A scanning electron microscope (SEM, Philips, XL20, Amsterdam, The Netherlands) was used to characterize the surface morphology of the PE. The PE samples were spread onto electric glue, which was fixed on a metallic base. Dried samples were coated with a thin layer of gold in vacuum. Then, temperature rising elution fractionation (TREF) was performed in a model 200+ instrument from Polymer Char S. A. (Valencia, Spain). Standard Conditions were used in all analyses, with $60 \mathrm{mg}$ in $20 \mathrm{~mL}$ of 1,2,4-trichlorobenzene (TCB), $0.3 \mathrm{~mL}$ analysis sample volume, crystallization rate of $0.5^{\circ} \mathrm{C} / \mathrm{min}$, and elution rate of $1{ }^{\circ} \mathrm{C} / \mathrm{min}$.

\section{Conclusions}

In summary, a facile approach to tuning the polyolefin molecular structure of ethylene/ $\alpha$-olefin copolymer by supported catalysts with specific pore structure was developed. The loading amounts of $\mathrm{Zr}$ and $\mathrm{Al}$ of the POPs-supported metallocene catalysts were highly dependent on the pore structure of the porous polymers. The ethylene/ $\alpha$-olefin copolymerization results show that the pore size and pore size distribution of the POP supports which were characterized by non-local density functional theory (NLDFT) simulation had a significant impact on the molecular chain growth and the molecular weight distribution. The IR, DSC, and TREF analyses revealed that the molecular chain structure of the ethylene / $\alpha$-olefin copolymers from different POPs and silica-supported metallocene catalysts exhibited similar but different branching degree, double bond content, and chemical composition distributions. Thus, minute differences of thermal and crystalline behavior were detected mainly by the active species scattered in different pore structures. Due to replicating effect, porous ethylene / $\alpha$-olefin copolymers with varied surface morphology were obtained from the POPs-supported metallocene catalysts with different pore structure. This new way of controlling the molecular structure of polyolefins would be practical and suitable for tailoring the properties of polyolefins for metallocene complexes. 
Acknowledgments: This work was financially supported by PetroChina Company Limited.

Author Contributions: Xiong Wang and Yongxiao Bai conceived and designed the experiments; Xiaoyu Han and Renwei Xu perfomed the experiments; Xiaoyu Han and Feng Ren analyzed the data; Xiong Wang wrote the paper.

Conflicts of Interest: The authors declare no conflict of interest.

\section{References}

1. Sauter, D.W.; Popoff, N.; Bashir, M.A.; Szeto, K.C.; Gauvin, R.M.; Delevoye, L.; Taoufik, M.; Boisson, C. The design of a bipodal bis(pentafluorophenoxy)aluminate supported on silica as an activator for ethylene polymerization using surface organometallic chemistry. Chem. Commun. 2016, 52, 4776-4779. [CrossRef] [PubMed]

2. Stürzel, M.; Mihan, S.; Mülhaupt, R. From Multisite Polymerization Catalysis to Sustainable Materials and All-Polyolefin Composites. Chem. Rev. 2016, 116, 1398-1433. [CrossRef] [PubMed]

3. Alt, H.G.; Köppl, A. Effect of the nature of metallocene complexes of group IV metals on their performance in catalytic ethylene and propylene polymerization. Chem. Rev. 2000, 100, 1205-1222. [CrossRef] [PubMed]

4. Michelotti, M.; Altomare, A.; Ciardelli, F.; Roland, E. Zeolite supported polymerization catalysts: Copolymerization of ethylene and $\alpha$-olefins with metallocenes supported on HY zeolite. J. Mol. Catal. Part A Chem. 1998, 129, 241-248. [CrossRef]

5. Jüngling, S.; Koltzenberg, S.; Mülhaupt, R. Propene homo- and copolymerization using homogeneous and supported metallocene catalysts based on $\mathrm{Me}_{2} \mathrm{Si}$ (2-Me-Benz[e]lnd) ${ }_{2} \mathrm{ZrCl}_{2}$. J. Polym. Sci. Part A Polym. Chem. 1997, 35, 1-8. [CrossRef]

6. Tisse, V.F.; Prades, F.; Briquel, R.; Boisson, C.; Mckenna, T.F.L. Role of silica properties in the polymerisation of ethylene using supported metallocene catalysts. Macromol. Chem. Phys. 2010, 211, 91-102. [CrossRef]

7. Kumkaew, P.; Wanke, S.E.; Praserthdam, P.; Danumah, C.; Kaliaguine, S. Gas-phase ethylene polymerization using zirconocene supported on mesoporous molecular sieves. J. Appl. Polym. Sci. 2003, 87, 1161-1177. [CrossRef]

8. Meshkova, I.N.; Kudinova, O.I.; Kovaleva, N.Y.; Grinev, V.G.; Ladygina, T.A.; Kiseleva, E.V.; Novokshonova, L.A. Effect of the zeolite support on the polymerization of propylene with immobilized ansa-zirconocene catalysts. Polym. Sci. Ser. B 2009, 51, 401-408. [CrossRef]

9. Heurtefeu, B.; Bouilhac, C.; Cloutet, E.; Taton, D. Polymer support of "single-site" catalysts for heterogeneous olefin polymerization. Prog. Polym. Sci. 2011, 36, 89-126. [CrossRef]

10. Huang, R.; Duchateau, R.; Koning, C.E.; Chadwick, J.C. Zirconocene Immobilization and Activation on $\mathrm{MgCl}_{2}$-Based Supports: Factors Affecting Ethylene Polymerization Activity. Macromolecules 2008, 41, 579-590. [CrossRef]

11. Upton, J.; Hlatky, G.G. Supported ionic metallocene polymerization catalysts. Macromolecules 1996, 29, 8019-8020.

12. Han, W.; Müller, C.; Vogt, D.; Niemantsverdriet, J.W.; Thüne, P.C. Introducing a Flat Model of the Silica-Supported Bis(imino)pyridyl Iron(II) Polyolefin Catalyst. Macromol. Rapid Commun. 2006, 27, 279-283. [CrossRef]

13. Moreira, S.C.; Maria de Fatima, V.M. Polyethylene synthesis using zeolite as support for metallocene catalyst. Eur. Polym. J. 2001, 37, 2123-2130. [CrossRef]

14. Matsui, S.; Mitani, M.; Saito, J.; Tohi, Y.; Makio, H.; Matsukawa, N.; Takagi, Y.; Tsuru, K.; Nitabaru, M.; Nakano, T.; et al. A family of zirconium complexes having two phenoxy-imine chelate ligands for olefin polymerization. J. Am. Chem. Soc. 2001, 123, 6847-6856. [CrossRef]

15. Czaja, K.; Korach, L. Synthesis and activity of zirconocene catalysts supported on silica-type sol-gel carrier for ethylene polymerization. Polym. Bull. 2001, 46, 175-182.

16. Vayá, V.I.; Belelli, P.G.; Santos, J.H.Z.; Ferreia, M.L.; Damiani, D.E. Influence of Acidic Support in Metallocene Catalysts for Ethylene Polymerization. J. Catal. 2001, 204, 1-10. [CrossRef]

17. Severn, J.R.; Chadwick, J.C.; Duchateau, R.; Friederichs, N. "Bound but not gagged" immobilizing single-site $\alpha$-olefin polymerization catalysts. Chem. Rev. 2005, 105, 4073-4147. [CrossRef] [PubMed]

18. Shen, J.; Xu, K.; Feng, L.; Wang, L.; Yuan, Y. A novel zirconocene/ultradispersed diamond black powder supported catalytic system for ethylene polymerization. Eur. Polym. J. 2002, 38, 2125-2128. 
19. Sacchi, M.C.; Zucchi, D.; Tritto, I.; Locatelli, P. Silica-supported metallocenes: Stereochemical comparison between homogeneous and heterogeneous catalysis. Macromol. Rapid Commun. 1995, 16, 581-590. [CrossRef]

20. Roscoe, S.B.; Fréchet, J.M.; Walzer, J.F.; Dias, A.J. Polyolefin spheres from metallocenes supported on noninteracting polystyrene. Science 1998, 280, 270-273. [CrossRef] [PubMed]

21. Nishida, H.; Uozumi, T.; Arai, T.; Soga, K. Polystyrene-supported metallocene catalysts for olefin polymerizations. Macromol. Rapid Commun. 1995, 16, 821-830. [CrossRef]

22. Hong, S.C.; Teranishi, T.; Soga, K. Investigation on the polymer particle growth in ethylene polymerization with PS beads supported rac- $\mathrm{Ph}_{2} \mathrm{Si}(\mathrm{Ind})_{2} \mathrm{ZrCl}_{2}$ catalyst. Polymer 1998, 39, 7153-7157. [CrossRef]

23. Dhanalaxmi, K.; Singuru, R.; Kundu, S.K.; Reddy, B.M.; Bhaumik, A.; Mondal, J. Strongly coupled Mn $\mathrm{Mn}_{3}$ $\mathrm{O}_{4}$-porous organic polymer hybrid: A robust, durable and potential nanocatalyst for alcohol oxidation reactions. RSC Adv. 2016, 6, 36728-36735. [CrossRef]

24. Mondal, J.; Kundu, S.K.; Ng, W.K.H.; Singuru, R.; Borah, P.; Hirao, H.; Zhao, Y.; Bhaumik, A. Fabrication of ruthenium nanoparticles in porous organic polymers: Towards advanced heterogeneous catalytic nanoreactors. Chem. Eur. J. 2015, 21, 19016-19027. [CrossRef] [PubMed]

25. Mondal, J.; Biswas, A.; Chiba, S.; Zhao, Y. $\mathrm{Cu}^{0}$ Nanoparticles Deposited on Nanoporous Polymers: A Recyclable Heterogeneous Nanocatalyst for Ullmann Coupling of Aryl Halides with Amines in Water. Sci. Rep. 2015, 5, 8294. [CrossRef] [PubMed]

26. Kaur, P.; Hupp, J.T.; Nguyen, S.T. Porous organic polymers in catalysis: Opportunities and challenges. ACS Catal. 2011, 1, 819-835. [CrossRef]

27. Bildirir, H.; Osken, I.; Ozturk, T.; Thomas, A. Reversible Doping of a Dithienothiophene-Based Conjugated Microporous Polymer. Chem. Eur. J. 2015, 21, 9306-9311. [CrossRef] [PubMed]

28. Gomes, R.; Bhaumik, A. Highly porous organic polymers bearing tertiary amine group and their exceptionally high $\mathrm{CO}_{2}$ uptake capacities. J. Solid State Chem. 2015, 222, 7-11. [CrossRef]

29. Wang, X.; Xu, R.; Zhu, B.; Li, Y.; Han, X. Metal oxide as a template in the preparation of porous poly (2-hydroxyethylmethylacrylate-co-divinylbenzene) particles as a metallocene catalyst support. RSC Adv. 2016, 6, 52464-52474. [CrossRef]

30. Wang, X.; Xu, R.; Zhu, B.; Li, Y.; Ma, Y. Synthesis and characterization of functional porous organic polymers as efficient metallocene catalyst supports. New J. Chem. 2016, 40, 8324-8333. [CrossRef]

31. Zhang, Y.; Riduan, S.N. Functional porous organic polymers for heterogeneous catalysis. Chem. Soc. Rev. 2012, 41, 2083-2094. [CrossRef] [PubMed]

32. Wang, X.; Li, Z.; Han, X.; Han, Z.; Bai, Y. Highly tunable porous organic polymer (POP) supports for metallocene-based ethylene polymerization. Appl. Surf. Sci. 2017, 420, 496-503. [CrossRef]

33. Kazatchkov, I.B.; Bohnet, N.; Goyal, S.K.; Hatzikiriakos, S.G. Influence of molecular structure on the rheological and processing behavior of polyethylene resins. Polym. Eng. Sci. 1999, 39, 804-815. [CrossRef]

34. Huang, W.; Sun, X.; Ma, H.; Huang, J. Ethylene homopolymerization and ethylene/1-hexene copolymerization catalysed by mixed salicylaldiminatocyclopentadienyl zirconium complexes. Inorg. Chim. Acta 2010, 363, 2009-2015. [CrossRef]

35. Bergamo, A.L.; Chagas, R.P.; Casagrande, O.L., Jr. Half-metallocene zirconium complex bearing tridentate $[\mathrm{N}, \mathrm{N}, \mathrm{O}]$ ligand and its use in homo-and copolymerization of ethylene. Catal. Commun. 2013, 42, $113-115$. [CrossRef]

36. Lei, J.; Li, D.; Wang, H.; Zhou, G. Porous polyethylene spheres with nanofiber structure from Ziegler-Natta catalyst supported on porous polymer particles. Polymer 2011, 52, 602-605.

37. Kageyama, K.; Tamazawa, J.; Aida, T. Extrusion polymerization: Catalyzed synthesis of crystalline linear polyethylene nanofibers within a mesoporous silica. Science 1999, 285, 2113-2115. [CrossRef] [PubMed]

38. Mays, J.W.; Puckett, A.D. Molecular Weight and Molecular Weight Distribution of Polyolefins. In Handbook of Polyolefins; Vasile, C., Seymour, R.B., Eds.; Marcel Dekker: New York, NY, USA, 1993; Volume 2, p. 133.

39. ASTM. Standard Test Methods for Absorbance of Polyethylene due to Methyl Groups at $1378 \mathrm{~cm}^{-1}$; ASTM D 2238-1992; ASTM International: West Conshohocken, PA, USA, 2012.

40. ASTM. Standard Test Method for Methyl (Comonmer) Content in Polyethylene by Infrared Spectrophotometry; ASTM D 6645-2001; ASTM International: West Conshohocken, PA, USA, 2010.

41. Xie, K.; Chen, D.; Cai, X.; Hou, B.; Zhang, Z. Study on the practical characterization of the microstructure of polyethylene by infrared spectroscopy. China Synth. Resin Plast. 2005, 22, 48-52. 
42. Kissin, Y.V.; Goldman, A.S. Chemistry and mechanism of alkene polymerization reactions with metallocene catalysts. Macromol. Chem. Phys. 2009, 210, 1942-1956. [CrossRef]

43. Yu, Y.; Tso, C.C.; DesLauriers, P.J. Molecular characteristics of room-temperature soluble fractions of low-density polyethylene film resins. J. Appl. Polym. Sci. 2006, 100, 4992-5006. [CrossRef]

44. Soares, J.B.P.; Mckenna, T.F. Polyolefin Microstructural Modeling. In Polyolefin Reaction Engineering; Wiley-VCH Verlag \& Co. KGaA: Weinheim, Germany, 2012; pp. 187-199, ISBN 978-3-527-64697-5.

(c)

(C) 2018 by the authors. Licensee MDPI, Basel, Switzerland. This article is an open access article distributed under the terms and conditions of the Creative Commons Attribution (CC BY) license (http://creativecommons.org/licenses/by/4.0/). 